Check for updates

Cite this: Chem. Commun., 2018, 54, 3997

Received 19th December 2017, Accepted 23rd March 2018

DOI: $10.1039 / c 7 c c 09708 e$

rsc.li/chemcomm

\section{Failure mechanisms of 2D silicon film anodes: in situ observations and simulations on crack evolution $\dagger$}

\author{
Le Yang, ${ }^{a}$ Hao-Sen Chen, (D) *b Hanqing Jiang, ${ }^{c}$ Yu-Jie Wei, ${ }^{d}$ Wei-Li Song (D) ${ }^{b}$ and \\ Dai-Ning Fang ${ }^{\mathrm{ab}}$
}

\begin{abstract}
An in situ optical system was used to observe the failure processes of two-dimensional silicon film anodes, suggesting a new debonding mode based on crack crushing. The stress evolution upon lithiation was quantitatively analyzed via fully coupled finite element simulations, confirming the crack crushing induced failure mechanisms in 2D silicon anodes.
\end{abstract}

Lithium-ion batteries (LIBs) of high energy density are desirable in many fields such as consumer electronics (cell phones, laptops, computers, etc.) and hybrid and electric vehicles. ${ }^{1}$ The rapid development in these fields demands higher capacity and enhanced durable LIBs. Silicon is one of the promising anode materials for high performance LIBs, since it possesses the highest theoretical specific capacity of $4200 \mathrm{~mA} \mathrm{~h} \mathrm{~g}^{-1}\left(\mathrm{Li}_{4.4} \mathrm{Si}\right){ }^{2}$ Nevertheless, its volume expansion can reach up to $\sim 300 \%$ after full lithiation, and this huge volume change during lithiation results in high mechanical stresses and massive cracking. ${ }^{3-5}$ Silicon electrodes, thus, usually show a shorter cycle life compared to the commercial graphite electrodes. A variety of silicon electrodes have been studied to accommodate this huge volume change, including silicon nanoparticles ${ }^{3,4}$ and two-dimensional (2D) silicon thin films, ${ }^{6-8}$ along with binder development of silicon anodes. ${ }^{9-11}$

The $e x$ situ experimental attempts ${ }^{6-8}$ and theoretical studies of diffusion-induced stress ${ }^{12-17}$ have found that the insertion of lithium ions into silicon films results in compressive stress in the film and the extraction of lithium results in tensile stress. If edges or cracks exist in the silicon films, the stress state will

\footnotetext{
${ }^{a}$ State Key Laboratory for Turbulence and Complex Systems,

College of Engineering, Peking University, Beijing, 100871, China

${ }^{b}$ State Key Laboratory of Explosion Science and Technology, Institute of Advanced Structure Technology, Beijing Institute of Technology, Beijing 100081, China. E-mail: chenhs@bit.edu.cn,weilis@bit.edu.cn

${ }^{c}$ School for Engineering of Matter, Transport and Energy, Arizona State University, Tempe, AZ 85287, USA

${ }^{d}$ LNM, Institute of Mechanics, Chinese Academy of Sciences, Beijing 100190, China $\dagger$ Electronic supplementary information (ESI) available. See DOI: 10.1039/ c7cc09708e
}

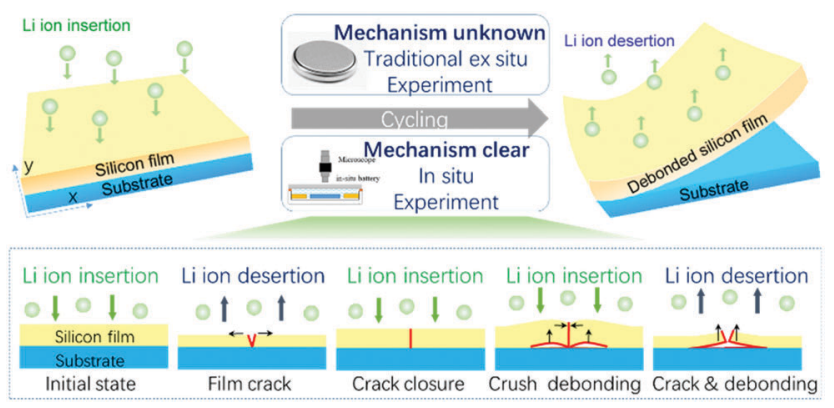

Fig. 1 Schematic illustration of silicon film crack and debonding during charge-discharge cycles. Mechanisms obtained from the traditional ex situ experiments are still not clear. Observation through the in situ experiment indicates that the electrode film has experienced film crack, crack closure, debonding due to crack face crushing and debonding during lithium insertion and desertion.

become very complicated. These stresses will lead to film cracks and interface debonding, as shown in Fig. 1 (top right). The mixed crack modes generated in 2D silicon film systems are known as the following: (a) channel cracks ${ }^{8,18-22}$ in the film during lithium extraction (cracking due to tensile stress $\sigma_{x x}$ ); (b) interface mode I debonding ${ }^{13,20,23-25}$ between silicon and the current collector because of lithium extraction induced tensile stress $\sigma_{y y}$; and (c) interface mode II debonding ${ }^{14,26,27}$ because of lithium insertion induced shear stress $\sigma_{x y}$. The new surface of the crack would cause capacity loss because of the generation of the solid electrolyte interphase (SEI) layer. The debonding between active materials and the current collector will reduce the electrical conductivity, and more importantly, it will lead to loss of active materials.

Despite these experiments and theoretical models that attempted to explain the failure mechanisms of 2D silicon films during charge-discharge cycles, there are still some puzzles on the failure evolution as charging time evolves due to the limitations of ex situ experiments. In this letter, in situ optical characterizations were performed by employing an electrochemical cell under an optical microscope to understand the failure 
mechanism of 2D silicon films during charge-discharge cycles. Based on such an in situ system, crack and debonding evolutions of a 2D silicon film electrode were observed. The entire failure evolution scheme is shown in Fig. 1 and the electrode film has mainly experienced four stages (bottom panel of Fig. 1), namely, film crack, crack closure, debonding due to crack face crushing, and debonding induced by lithium extraction. In addition to the known modes (i.e., film cracking and crack closure), a new debonding mode (debonding due to crack face crushing) was found. A fully coupled finite element simulation was used to study the stress state of the new debonding mode.

Experimentally, quartz wafers $(500 \mu \mathrm{m}$ thick, double-side polished) were used as substrates for electrode fabrication. The $\sim 300 \mathrm{~nm} \mathrm{Cu}$ current collectors and $311 \mathrm{~nm}$ thick (Fig. S1, ESI $\dagger$ ) silicon electrodes were deposited in a commercial magnetron sputtering system. The crystal structure of the as-deposited film was investigated using XRD (X'PERT PRO). The chemical state of the Si films was analyzed using XPS (PHI Quantera II SXM). The initial-state and final-state morphological changes were imaged using a field-emission scanning electron microscope (SEM, FEI Quanta 250). An optical microscope (Keyence Corporation) was used to in situ characterize the continuous morphological changes of the 2D silicon films (Fig. S2, ESI $\dagger$ ). The home-made in situ electrochemical cell (details in Fig. S3, ESI $\dagger$ ) was assembled in a high-purity argon filled glove box (Mbraun Inc.) The galvanostatic current densities were 60 and $30 \mu \mathrm{A} \mathrm{cm}^{-2}$ for the discharge and charge, respectively. The cycle voltage was in the range of 1.5-0.01 V (vs. $\left.\mathrm{Li} / \mathrm{Li}^{+}\right)$.

Fig. 2a shows the XRD patterns of the as-deposited Si films. No diffraction peaks assigned to crystalline Si were observed. These results confirm that the Si film is amorphous. Fig. $2 \mathrm{~b}$ shows the XPS O1s and Si2p spectra of the as-deposited Si films after Ar ion etching for $3 \mathrm{~min}$ and $6 \mathrm{~min}$ (etching speed is about $1 \mathrm{~nm} \min ^{-1}$ for crystal silicon). The peak at $\sim 99.2 \mathrm{eV}$ can be associated with pure silicon. The O1s spectrum at $\sim 532 \mathrm{eV}$ indicates that the films were slightly oxidized during spurting. XPS elemental analysis was performed to investigate the number of $\mathrm{O}$ and $\mathrm{Si}$, and the result shows that the average atomic concentrations of $\mathrm{O}$ and $\mathrm{Si}$ are $3.6 \%$ and $96.3 \%$, respectively.

Fig. $2 \mathrm{c}-\mathrm{f}$ shows the electrochemical characterization and ex situ SEM images of silicon films after three cycles, suggesting the coulombic efficiency of silicon electrodes is just $15.16 \%$ and $55.62 \%$ for the first and second cycles, respectively. The SEM image (Fig. 2f) shows plenty of cracks and debonding areas after several cycles. This seems to indicate that debonding is a key factor in the degradation of battery performance.

The in situ optical experiments show the entire failure processes in electrochemical reactions. Fig. 3a-f shows the optical images and schemes of the 2D silicon electrodes, corresponding to the points A-F in the voltage curve plotted in Fig. 2d. During the first lithium insertion (Fig. 3a and b, discharge for half-cell), no significant change was observed on the surface morphology of the silicon films, demonstrating that the interfacial strength is sufficiently strong to avoid buckling (the normal interface stress $\sigma_{y y}$ is very small for the unbroken film, Fig. S5, ESI $\dagger$ ) and thus lithium insertion only increased the a

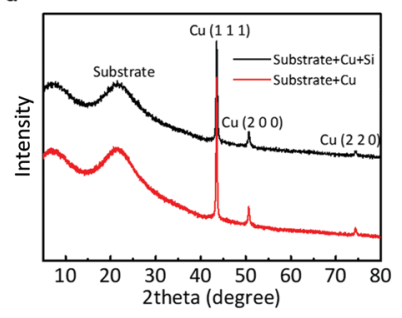

b
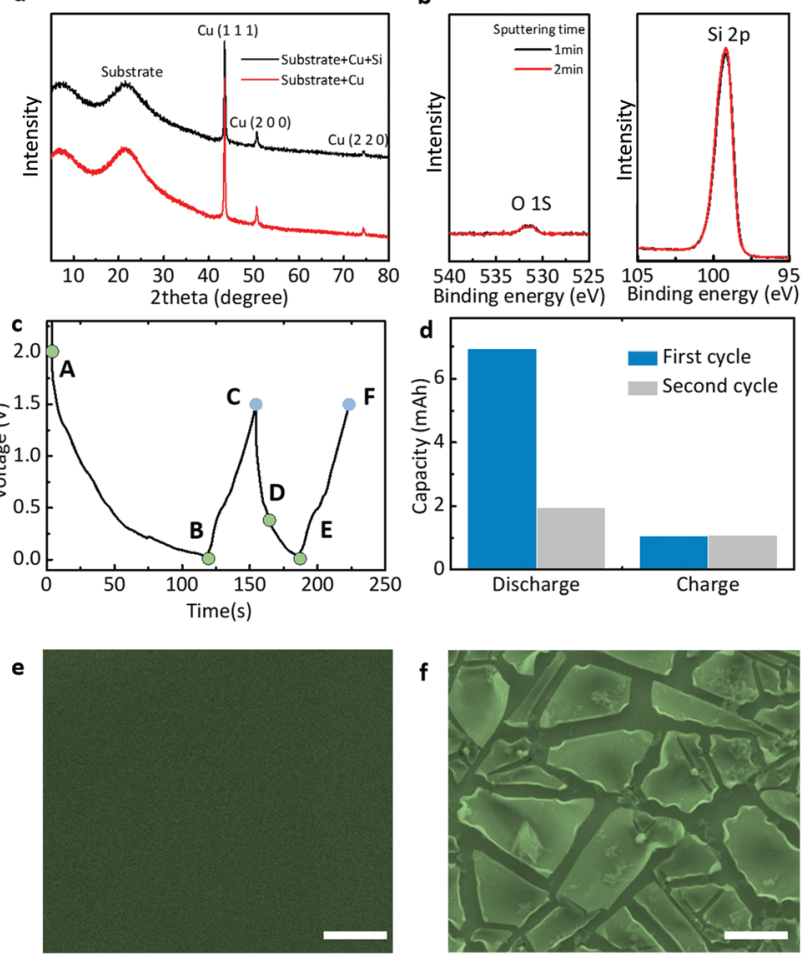

Fig. 2 (a) XRD patterns of $\mathrm{Cu}$ and Si/Cu films on a quartz substrate. (b) XPS spectra of O1s and Si2p of Si film at a depth after Ar ion etching for 3 min and $6 \mathrm{~min}$. (c) A voltage versus time plot; the mark points correspond to the optical micrographs in Fig. 3, and (d) charge and discharge capacity. The SEM image of the silicon film before (e) and after three charge-discharge cycles (f). The scale bare is $20 \mu \mathrm{m}$.

thickness of the silicon films. After the first lithium desertion (Fig. 3c), mode I channel cracks appeared in the film due to the tensile stress in the film, splitting the silicon film into many silicon islands. These in-plane cracks would stop propagating until the silicon islands became small enough. As aforementioned, the capacity loss induced by the mode I crack only occurs when a new surface is generated, leading to new SEI formation. If there are only stable cracks inside the film, the capacity will remain constant. The rate capability might be even improved sometimes when the surface area of the silicon films is higher than that of the initial state. These in-plane cracks will act as initial cracks during the subsequent cycles. The following analysis will show that the debonding induced by initial cracks is more serious than the cracks.

Fig. 3d-f (and Video S1 in the ESI $\dagger$ ) show the debonding phenomena due to the interaction of silicon island edges, namely, the initial crack surfaces. First, the crack closure was observed during the second discharge to $0.38 \mathrm{~V}$ (Fig. 3d), which resulted from the lithium insertion induced expansion. Then, the crushing induced interface debonding appeared upon gradual insertion of lithium ions. The black region around the cracks (Fig. 3e) indicates the debonding between the silicon film and the current collector. This debonding phenomenon has been rarely reported previously, which corresponds to a new debonding mode, i.e. debonding due to crack surface crushing. During lithium extraction 

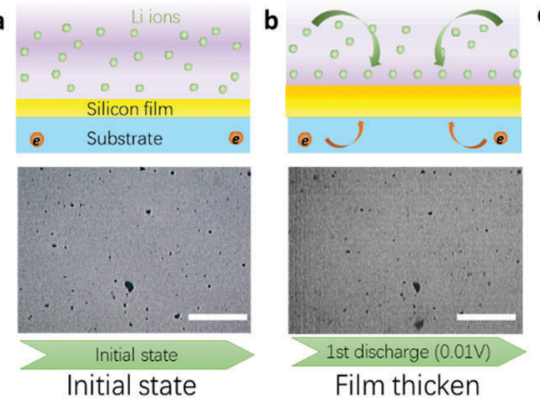

Film thicken

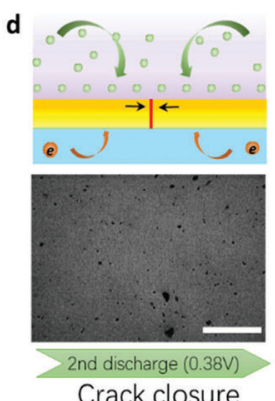

d

Crack closure

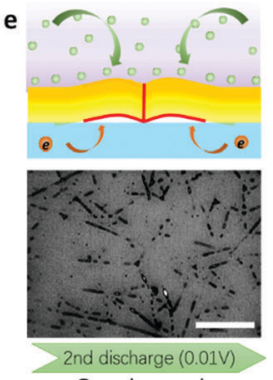

Crack crush
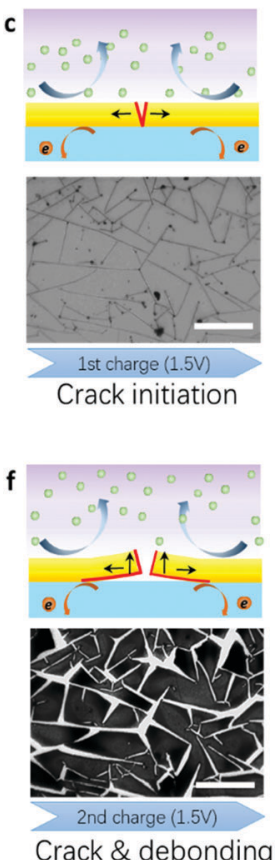

Fig. 3 The initial film (a) has experienced film expansion (b), film crack (c), crack closure (d), debonding due to crack face crushing (e) and mode I debonding during lithium desertion (f). The scale bare is $20 \mu \mathrm{m}$.

(Fig. 3f), large area debonding (mode II debonding) ultimately appeared in the initial debonding areas. The SEM image (Fig. 2d) shows the same debonding area and demonstrates that the black areas in the optical images refer to the debonding areas as well.

From the experimental results aforementioned, i.e., the new debonding phenomenon, debonding induced by crack face crushing, is a critical process of the electrode failure. It is thus necessary to understand the mechanisms of the crushing induced interface debonding. Fig. 4a shows the scheme of boundary conditions, corresponding to the mode II debonding and the crushing induced debonding. The substrate (i.e., copper current collector) was fixed, and the lithium is inserted from the surfaces of the silicon film and generated cracks. For the mode II debonding, due to the constraint of the substrate and expansion of the silicon film, the source of the debonding is apparently shear stress $\sigma_{x y}$. For crushing induced debonding, the stress state can be more complicated. Qualitatively, the initial contact of two crack surfaces could appear at the surface of silicon islands, and therefore the compressive stress $\sigma_{x x}$ from the crack surface would result in a bending deformation. Such a bending deformation will generate a tensile stress $\sigma_{y y}$ at the interface, causing crush debonding. These interface debonding mechanisms are represented in Fig. 4 b. The weak $\mathrm{Cu}-\mathrm{Si}$ interface is insufficient to resist the shearing stress or the tensile stress, leading to interface debonding.

For quantitative analysis, finite element simulation was performed. In a lithiated silicon, the deformation gradient $\boldsymbol{F}$ could be decomposed into elasticity, plasticity and concentration parts and thus the principal stretches can be also decomposed into three parts, ${ }^{24}$

$$
\lambda_{i}=\lambda_{i}^{\mathrm{e}} \lambda_{i}^{\mathrm{p}} \lambda_{i}^{\mathrm{c}}
$$
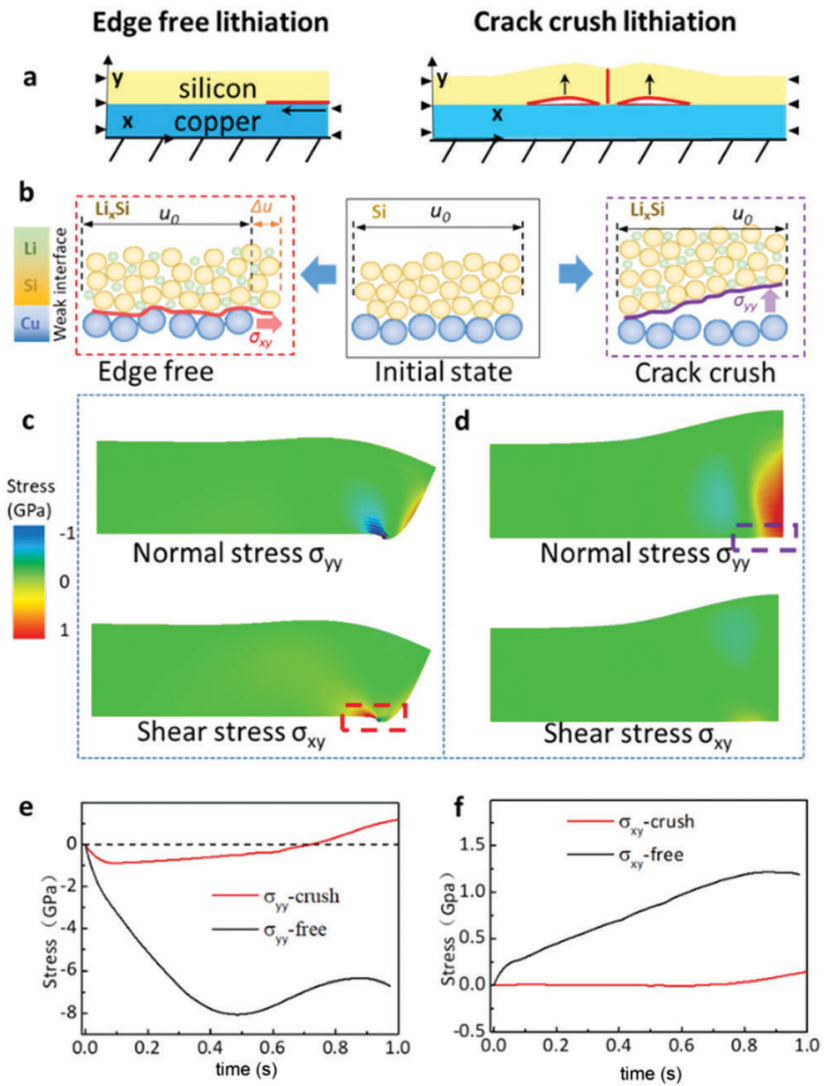

Fig. 4 (a) The boundary conditions of mode II debonding and debonding due to crack crushing during lithium insertion, and (b) debonding mechanisms under both boundaries conditions at the atomic scale. (c and d) The fields of normal stress and shear stress for two conditions are given by the simulation results after full lithiation. (e and $f$ ) The evolution of the stress at the right bottom point (the crack bottom).

where the superscripts 'e', 'p' and 'c' denote elastic, plastic and concentration parts, respectively, and here $i=1,2,3$, no summation convention. Then, the constitutive relationship including diffusion-induced deformation can be expressed as

$$
\sigma_{i}=\frac{1}{\lambda_{1} \lambda_{2} \lambda_{3}} \frac{\partial W}{\partial \ln \lambda_{i}^{\mathrm{e}}},
$$

where $W$ is the nominal free energy density of the system.

The chemical potential with the effect of stress can be expressed as

$$
\mu=\frac{\partial W}{\partial C}-\lambda_{1} \lambda_{2} \lambda_{3} \sigma_{\mathrm{h}} \frac{3 \mathrm{~d}}{\lambda^{\mathrm{c}}} \frac{\lambda^{\mathrm{c}}}{\mathrm{d} C}
$$

where $\sigma_{\mathrm{h}}=\frac{1}{3}\left(\sigma_{1}+\sigma_{2}+\sigma_{3}\right)$ is the hydrostatic stress.

A specific form of the chemical energy density $W$ is given by the following: ${ }^{28}$

$$
W=R T C_{\max }(\bar{C} \ln \bar{C})+(1-\bar{C}) \ln (1-\bar{C}),
$$

where $C_{\max }$ is the maximum Li concentration, $\bar{C}=C / C_{\max }$ is the normalized nominal Li concentration in silicon, and $R T$ is the product of gas constant $R$ and absolute temperature $T$. 
The detailed derivation can be found in the work by Zhao et $a{ }^{29}{ }^{29} \mathrm{~A}$ fully coupled large deformation and diffusion finite element simulation using ABAQUS user-defined subroutines were performed; the involved subroutines are provided in the ESI. $\dagger$ The simulation details of coupled electro-chemical reaction processes were described in our previous studies. ${ }^{30}$ The substrate quartz is assumed to be rigid, and the $\mathrm{Cu}$ current collector is assumed to be elastic. The details of the finite element analysis (FEA) model can be found in Fig. S4 (ESI $\dagger$ ). The parameters used in the simulation are given in Table $\mathrm{S} 1$ (ESI $\dagger$ ).

The stress states of mode I and mode II debonding in the lithiation process were simulated. The final stress states after full lithiation are given in Fig. $4 \mathrm{c}$ and $\mathrm{d}$. The normal stress $\sigma_{y y}$ and shear stress $\sigma_{x y}$ of the point at the right bottom (i.e. the crack bottom) during lithiation are given in Fig. 4e and f, corresponding to the two modes. Fig. $4 \mathrm{~d}$ shows that the interface normal stress $\sigma_{y y}$ is the major reason of the crushing induced debonding. A comparison between Fig. 4e and $\mathrm{f}$ (the free edge lithiation mode) suggests that this interface normal stress is obviously generated because of crack surface crushing. Fig. 4e shows that the normal stress of the crushing induced interface is changed from the compressive stress to the tensile stress, compared with the free edge lithiation mode, and the tensile stress will still rise during lithiation. Furthermore, Fig. 4d shows that the crushing weakens the interface shear stress $\sigma_{x y}$, which indicates the debonding mode change due to crushing, also shown in Fig. 4f. From the comparison between these two modes, both the shear stress and normal stress will cause the interface debonding; however, there are many cracks inside the active materials rather than the free edge. Thus, the crushing induced debonding is the major reason for anode degradation.

In summary, a new failure mechanism of $2 \mathrm{D}$ silicon film electrodes was revealed by in situ optical experiments. The new debonding mode, crushing induced debonding, was identified as the key issue for electrode failure. Subsequently, the stress state was quantitatively analyzed using a fully coupled finite element method, consistent with the experimental results. Furthermore, the results suggest that the inner crack crushing is a very important reason for battery performance degradation, which should be studied deeply. Fully understanding the crack crushing mechanism would be very useful for the rational design of stable LIBs with high energy density.

The authors are grateful for the support by the National Natural Science Foundation of China (grant No. 11672341, No. 111572002, and No. 51302011) and the 973 Project (No. 2015CB932500). Support by the Foundation for Innovative Research Groups of the National Natural Science Foundation of China (No. 11521202) is also acknowledged. The work was also financially supported by the National Materials Genome Project (2016YFB0700600) and Beijing Natural Science Foundation (Grant No. 16L00001).

\section{Conflicts of interest}

There are no conflicts to declare.

\section{Notes and references}

1 M. Armand and J. M. Tarascon, Nature, 2008, 451, 652-657.

2 X. Su, Q. Wu, J. Li, X. Xiao, A. Lott, W. Lu, B. W. Sheldon and J. Wu, Adv. Energy Mater., 2014, 4, 1300882.

3 L. Luo, J. Wu, J. Luo, J. Huang and V. P. Dravid, Sci. Rep., 2014, 4, 4322 .

4 M. T. McDowell, I. Ryu, S. W. Lee, C. Wang, W. D. Nix and Y. Cui, Adv. Mater., 2012, 24, 6034-6041.

5 J. Lang, B. Ding, T. Zhu, H. Su, H. Luo, L. Qi, K. Liu, K. Wang, N. Hussain, C. Zhao, X. Li, H. Gao and H. Wu, Adv. Mater., 2016, 28, 10236-10243.

6 V. A. Sethuraman, M. J. Chon, M. Shimshak, N. Van Winkle and P. R. Guduru, Electrochem. Commun., 2010, 12, 1614-1617.

7 M. Pharr, Y. S. Choi, D. Lee, K. H. Oh and J. J. Vlassak, J. Power Sources, 2016, 304, 164-169.

8 M. Pharr, Z. Suo and J. J. Vlassak, Nano Lett., 2013, 13, 5570-5577.

9 Y. K. Jeong, T. Kwon, I. Lee, T. Kim, A. Coskun and J. W. Choi, Energy Environ. Sci., 2015, 8, 1224-1230.

10 M. T. Jeena, J. Lee, S. H. Kim, C. Kim, J. Kim, S. Park and J. Ryu, ACS Appl. Mater. Interfaces, 2014, 6, 18001-18007.

11 S. Lim, K. Lee, I. Shin, A. Tron, J. Mun, T. Yim and T. Kim, J. Power Sources, 2017, 360, 585-592.

12 F. Yang, J. Power Sources, 2011, 196, 465-469.

13 M. Liu, Int. J. Solids Struct., 2015, 67-68, 263-271.

14 M. Liu, J. Power Sources, 2015, 275, 760-768.

15 H. Haftbaradaran, J. Power Sources, 2015, 288, 278-287.

16 S. K. Soni, B. W. Sheldon, X. Xiao, M. W. Verbrugge, D. Ahn, H. Haftbaradaran and H. Gao, J. Electrochem. Soc., 2012, 159, A38-A43.

17 B. Lu, Y. Song and J. Zhang, J. Power Sources, 2015, 289, 168-183.

18 L. Y. Beaulieu, K. W. Eberman, R. L. Turner, L. J. Krause and J. R. Dahn, Electrochem. Solid-State Lett., 2001, 4, A137-A140.

19 J. Li, A. K. Dozier, Y. Li, F. Yang and Y. Cheng, J. Electrochem. Soc., 2011, 158, A689-A694.

20 J. P. Maranchi, A. F. Hepp, A. G. Evans, N. T. Nuhfer and P. N. Kumta, J. Electrochem. Soc., 2006, 153, A1246-A1253.

21 Y. H. Wang, Y. He, R. J. Xiao, H. Li, K. E. Aifantis and X. J. Huang, J. Power Sources, 2012, 202, 236-245.

22 C. Yu, X. Li, T. Ma, J. Rong, R. Zhang, J. Shaffer, Y. An, Q. Liu, B. Wei and H. Jiang, Adv. Energy Mater., 2012, 2, 68-73.

23 L. Y. Beaulieu, V. K. Cumyn, K. W. Eberman, L. J. Krause and J. R. Dahn, Rev. Sci. Instrum., 2001, 72, 3313-3319.

24 X. Xiao, P. Liu, M. W. Verbrugge, H. Haftbaradaran and H. Gao, J. Power Sources, 2011, 196, 1409-1416.

25 Y. He, X. Yu, G. Li, R. Wang, H. Li, Y. Wang, H. Gao and X. Huang, J. Power Sources, 2012, 216, 131-138.

26 S. Pal, S. S. Damle, S. H. Patel, M. K. Datta, P. N. Kumta and S. Maiti, J. Power Sources, 2014, 246, 149-159.

27 H. Haftbaradaran, Int. J. Solids Struct., 2016, 91, 157-168.

28 H. Haftbaradaran, J. Song, W. A. Curtin and H. Gao, J. Power Sources, 2011, 196, 361-370.

29 K. Zhao, M. Pharr, S. Cai, J. J. Vlassak and Z. Suo, J. Am. Ceram. Soc., 2011, 94, s226-s235.

30 Y. An and H. Jiang, Modell. Simul. Mater. Sci. Eng., 2013, 21, 0740077. 